\title{
É seguro atirar para cima? Uma analise da letalidade de projéteis subsônicos
}

\author{
Is it safe to shoot up? An analysis of the lethality of subsonic projectiles
}

\author{
Saulo Luis Lima da Silva*1@, Herman Fialho Fumiã² \\ ${ }^{1}$ Centro Federal de Educacao Tecnologica de Minas Gerais, Departamento de Formação Geral, Nepomuceno, MG, Brasil \\ ${ }^{2}$ Universidade do Estado de Minas Gerais, Carangola, MG, Brasil
}

\begin{abstract}
Recebido em 13 de Setembro, 2018. Revisado em 13 de Novembro, 2018. Aceito em 15 de Novembro, 2018.
Os tiros de comemoração referem-se aos tiros dados para o alto por ocasião de celebrações festivas. Este é um hábito em países como o Afeganistão, Paquistão e algumas regiões da América Latina. Neste artigo, resolvemos o problema de projéteis com velocidade subsônica atirados para alto, considerando a resistência do ar. Com fins didáticos, traçamos um paralelo entre esse caso e aquele em que a resistência do ar é desprezada. Concluímos que mesmo projéteis de pequeno calibre podem ser letais na queda, apesar da diminuição da velocidade e da energia cinética.
\end{abstract}

Palavras-chave: projéteis, letalidade, força de arrasto, resistência do ar

\begin{abstract}
Celebratory gunfire is the shooting of a firearm into the air in celebration. This is common in countries as Afghanistan, Pakistan and regions of Latin American. In this article, we have solved the problem of a projectile with subsonic speed shooted upward, considering the air resistance. For didactic purposes, we draw a parallel between the case with and without air resistance. We conclude that even small-caliber projectile can be lethal when falling, a despite the decrease in speed and kinetics energy.

Keywords: bullet, lethality, drag, air resistance
\end{abstract}

\section{Introdução}

Ocasionalmente vemos celebrações em filmes ou noticiários que envolvem pessoas atirando para o alto. Esse é um hábito culturamente aceito em diversas regiões do mundo como o Afeganistão, Paquistão, Índia e algumas partes da América Latina [1]. Apesar da sua associação com situações festivas, como celebrações do Ano Novo, Natal, casamentos, e etc., seria essa uma prática segura para as pessoas presentes nessas comemorações ou próximas das mesmas?

Se nos esquecermos por um momento da expressão "despreze a resistência do ar", tão utilizada nos exercícios de mecânica básica, a resposta será óbvia; a velocidade da bala na descida será a mesma que na subida e, consequentemente, as pessoas atingidas podem morrer. Contudo, como a resistência do ar aumenta com a velocidade e projéteis de armas movidos a expansão de gases possuem velocidades elevadas, a resistência do ar não pode ser desprezada nessas circunstâncias nem mesmo como aproximação.

Nesse artigo, resolvemos o problema de projéteis subsônicos atirados na vertical considerando a resistência do ar e analisamos a questão da possível letalidade desse projétil nessa situação. Essa discussão também possui

*Endereço de correspondência: saulolimasilva@yahoo.com.br. um forte apelo didático, uma vez que a resistência do ar é geralmente negligenciada nos cursos de mecânica básica. Por esse motivo, traçamos alguns paralelos entre os resultados apresentados ao se considerar e ao se desprezar a resistência do ar.

O trabalho está estruturado da seguinte forma: na seção II apresentamos os aspectos essenciais da força de arrasto para a nossa analise. A seção III é dedicada à análise do movimento vertical, tanto ascendente quanto descendente, do projétil. Os resultados e discussões são apresentados na seção IV. Na seção V apresentamos a conclusão.

\section{A força de arrasto}

A força de arrasto sobre um corpo imerso em um fluido e que se movimenta com uma velocidade $v$ em relação a esse fluido, pode ser descrita pela equação 1 .

$$
F_{a}=\frac{1}{2} \rho C_{d} A v^{2}
$$

em que $v$ é a velocidade do corpo em relação ao fluido, $\rho$ é a densidade do fluido, $C_{d}$ é o coeficiente de arrasto, e $A$ é a área de seção transversal do corpo. Essa forma funcional é adequada apenas para velocidades situadas entre alguns poucos metros por segundo e próximas à velocidade do som. 
A equação 1 admite como hipótese que o coeficiente de arrasto $C_{d}$ é constante. Essa é uma aproximação razoável em duas condições: quando o projétil se movimenta com uma velocidade abaixo da velocidade do som no meio e para projéteis que se movimentam em velocidades supersônicas, maiores que Mach 2 3 5]. Naturalmente, nosso tratamento não pode se aplicar a projéteis supersônicos, uma vez que nessa faixa de velocidades há grande variação no coeficiente de arrasto $C_{d}$, conforme podemos ver na (Figura 1). Portanto, nos limitaremos a projéteis subsônicos.

Projéteis trafegando a velocidades subsônicas tipicamente são aqueles atirados por revólveres e pistolas. Na Tabela 1, apresentamos a velocidade inicial $v_{0}$, ou seja, a velocidade de saída do cano da arma, para alguns calibres tipicamente utilizados em revólveres e pistolas. Também é apresentado a massa $m$ desses projéteis.

O coeficiente de arrasto $C_{d}$ é função da forma do projétil. Os projéteis da Tabela 1 possuem uma forma similar àquela do modelo padrão G1 (Figura 1). Como podemos ver da Figura 1] na região subsônica destacada, o coeficiente de arrasto médio é de 0,24. Adotaremos esse valor para o coeficiente de arrasto de todos os projéteis apresentados na Tabela 11 A título de comparação, observe a Figura 2 onde observamos resultados para o comportamento do coeficiente de arrasto de um projétil calibre .22

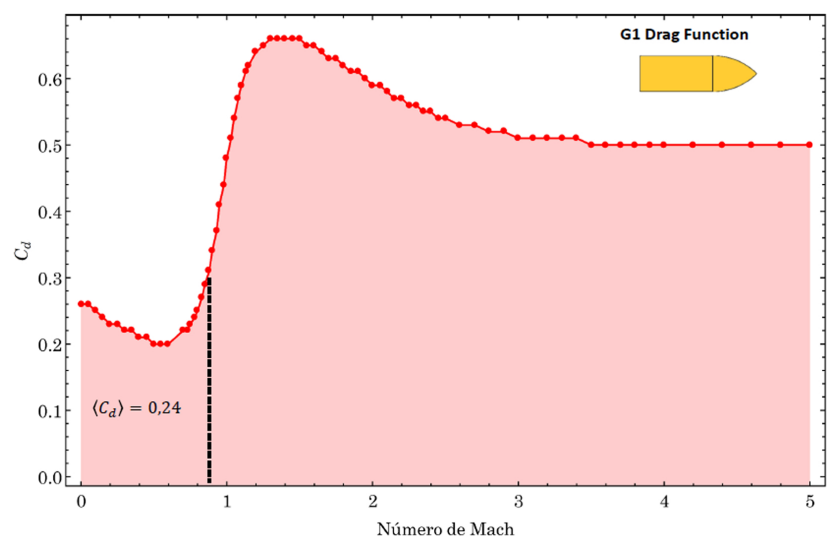

Figura 1: Coeficiente de arrasto em função do número de Mach para o modelo padrão de projétil do tipo G1. Figura produzida a partir de dados obtidos em [2].

Tabela 1: Massa $m$ e velocidade inicial $v_{0}$ de uma seleção de projéteis de pistolas e revólveres [6].

\begin{tabular}{lcc}
\hline Calibre & $m(\mathbf{g})$ & $v_{0}(\mathbf{m} / \mathbf{s})$ \\
\hline .22 short (5.6 mm short) & 1,8 & 290 \\
.22 long rifle & 1,8 & 330 \\
$6.35 \mathrm{~mm}$ Browning & 3,2 & 230 \\
$7.65 \mathrm{~mm}$ Browning & 4,7 & 300 \\
$9 \times 18 \mathrm{~mm}$ Police & 6,1 & 300 \\
$7.62 \mathrm{~mm}$ Nagant & 7,0 & 290 \\
.32 Smith and Wesson & 5,5 & 200 \\
.38 Smith and Wesson & 9,4 & 210 \\
.38 Smith and Wesson special & 10,2 & 260 \\
.45 Automatic Colt Pistol & 14,9 & 260 \\
\hline
\end{tabular}

long rifle como função do número de Mach. Note que os valores do coeficiente de arrasto e o formato do projétil estão em um acordo razoável com o do modelo G1.

\section{Movimento de projéteis na vertical}

No intuito de estudar o movimento de projéteis atirados para cima, na vertical, vamos dividi-lo em duas partes: a subida (vertical ascendente) e a descida (vertical descendente).

Adotamos a localização de onde o projétil sai do cano da arma como sendo a origem $y=0$ do nosso sistema de coordenadas e adotamos o tempo em que o projétil é atirado como $t=0$.

\subsection{Vertical ascendente}

No caso do movimento ascendente, a segunda lei de Newton nos permite escrever (em uma dimensão)

$$
m \frac{d v}{d t}=-\left(F_{d}+P\right)=-\left(\frac{1}{2} \rho A C_{d} v^{2}+m g\right),
$$

onde $m$ é a massa do projétil, $P$ é o peso do mesmo e $g$ é a aceleração da gravidade.

A equação 2 é separável e pode ser integrada de forma trivial. A posição e velocidade do projétil em função do tempo (tomando os outros parâmetros como constantes) no movimento ascendente do projétil são dadas, respectivamente, por:

$$
y(t)=\frac{v_{t}^{2}}{g} \ln \left|\frac{\cos \left[\frac{g}{v_{t}}\left(t_{s}-t\right)\right]}{\cos \left(\frac{g t_{s}}{v_{t}}\right)}\right|, 0 \leq t \leq t_{s}
$$


e

$$
v(t)=v_{t} \tan \left[\arctan \left(\frac{v_{0}}{v_{t}}\right)-\frac{g}{v_{t}} t\right], 0 \leq t \leq t_{s}
$$

onde $v_{0}$ é a velocidade inicial do projétil ao sair da boca do cano da arma e $v_{t}$ é a velocidade atingida pelo projétil quando o peso e a força de arrasto se igualam, conhecida como velocidade terminal. A velocidade terminal pode ser obtida facilmente fazendo $\mathcal{D} v_{t}^{2}=m g$, o que nos dá $v_{t}=\sqrt{\frac{m g}{\mathcal{D}}}$, em que $\mathcal{D}=\frac{1}{2} \rho A C_{d}$. Além disso, $t_{s}$ é o tempo que o projétil leva para atingir o ponto mais alto de sua trajetória. Esse tempo pode ser obtido fazendo $\left(v\left(t_{s}\right)=0\right)$ na equação (4). Com isso, obtemos

$$
t_{s}=\frac{v_{t}}{g} \arctan \left(\frac{v_{0}}{v_{t}}\right) .
$$

A altura máxima $h_{\max }$ atingida pelo projétil corresponde à posição $y\left(t_{s}\right)$. A expressão é dada por:

$$
h_{\max }=-\frac{v_{t}^{2}}{g} \ln \left|\cos \left(\frac{g t_{s}}{v_{t}}\right)\right| .
$$

O balanço de energia para esse caso também é bem simples de se fazer, uma vez que a energia (cinética) do projétil ao sair da boca do cano da arma é $k_{0}=(1 / 2) m v_{0}^{2}$ e ao atingir o ponto mais alto de sua trajetória sua energia (potencial gravitacional) será ${ }^{1} U_{g}=m g h_{\max }$. Esses resultados nos permitem calcular facilmente a energia mecânica dissipada pela força não conservativa (a força de arrasto) durante a subida $\Delta E_{m s u b}=k_{0}-U_{g}$. Contudo, mais interessante para o nosso caso é a energia mecânica dissipada durante todo o trajeto. Que pode ser obtida através de $\Delta E_{m}=k_{0}-k_{q}$, em que $k_{q}=(1 / 2) m v_{q}^{2}$ é a energia cinética do projétil quando chega ao solo.

\subsection{Vertical descendente}

No caso do movimento descendente, a segunda lei de Newton pode ser escrita na forma

${ }^{1}$ Estamos considerando o nível da boca do cano da arma como um referencial onde adotamos a energia potencial gravitacional como sendo nula. Salientamos que no ponto mais alto da trajetória a velocidade do projétil será nula, portanto a energia cinética e a força de arrasto também serão.

$$
m \frac{d v}{d t}=F_{d}-m g=\frac{1}{2} \rho A C_{d} v^{2}-m g,
$$

de onde podemos obter a posição e velocidade do projétil em função do tempo. Fazendo a integração em (7), obtemos:

$$
y(t)=h_{\max }-\frac{v_{t}^{2}}{g} \ln \left[\cosh \left(\frac{g}{v_{t}}\left(t_{s}-t\right)\right)\right], t \geq t_{s}
$$

$$
v(t)=v_{t} \tanh \left(\frac{g}{v_{t}}\left(t_{s}-t\right)\right), t \geq t_{s} .
$$

O tempo de descida $t_{d}$ é obtido por meio da equação 8 impondo a condição $y\left(t_{d}\right)=0$. A expressão para o tempo de descida $t_{d}$ é:

$$
t_{d}=t_{s}-\frac{v_{t}}{g} \operatorname{arccosh}\left(\left|\cos \left(\frac{g t_{s}}{v_{t}}\right)\right|^{-1}\right) .
$$

\section{Resultados e Discussões}

\subsection{Tempos de subida e descida, altura máxima e velocidades terminais e de queda}

Desconsiderando a resistência do ar, os tempos de subida $t_{s}$ e queda $t_{q}$ para um mesmo projétil são iguais (na Tabela 2 $t_{s}=t_{q}=t_{0}$ ). Contudo, a situação com resistência do ar é bem diferente. Observe na Tabela 2 , por exemplo, que o tempo de queda $t_{q}$ para um projétil .22 long rifle é aproximadamente 1,67 vezes maior do que o tempo de subida $t_{s}$. De fato, isso ocorre porque na subida as forças de resistência do ar e o peso estão no mesmo sentido, enquanto na descida estão em sentidos contrários.

É interessante compararmos os tempos para o caso com e sem a resistência do ar (Tabela 2). Por exemplo, para o calibre $7.65 \mathrm{~mm}$ Browning, desconsiderando a resistência do ar, o tempo de subida ou descida $t_{0}$ é de $30,6 \mathrm{~s}$, o que nos dá um tempo total de 61,2 s. Quando a resistência do ar é levada em consideração, observamos que o tempo total $t_{\text {total }}$ é de apenas $27,2 \mathrm{~s}$, ou seja, o famoso despreze a resistência do ar seria tão inadequado nesse caso que

Tabela 2: Tempos de subida $t_{s}$, descida $t_{d}$, total $t_{\text {total }}$ e a razão $t_{d} / t_{s}$ para diversos projéteis considerando a resistência do ar. $\mathrm{O}$ tempo $t_{0}$ corresponde ao tempo de subida ou descida para o caso em que a resistência do ar é ignorada.

\begin{tabular}{lccccc}
\hline Calibre & $t_{s}$ & $t_{d}$ & $t_{\text {total }}$ & $t_{d} / t_{s}$ & $t_{0}$ \\
& $(\mathrm{~s})$ & $(\mathrm{s})$ & $(\mathrm{s})$ & & $(\mathrm{s})$ \\
\hline .22 short (5.6 mm short) & 9,4 & 15,0 & 24,4 & 1,61 & 29,6 \\
.22 long rifle & 9,6 & 15,9 & 25,5 & 1,67 & 33,7 \\
6.35 mm Browning & 10,2 & 14,6 & 24,9 & 1,43 & 23,5 \\
7.65 mm Browning & 10,6 & 16,5 & 27,2 & 1,55 & 30,6 \\
9x18 mm Police & 10,3 & 13,9 & 24,1 & 1,36 & 20,4 \\
7.62 mm Nagant & 10,7 & 16,6 & 27,3 & 1,55 & 30,6 \\
.32 Smith and Wesson & 12,4 & 18,0 & 30,4 & 1,45 & 29,6 \\
.38 Smith and Wesson & 11,4 & 15,1 & 26,4 & 1,33 & 21,4 \\
.38 Smith and Wesson special & 12,5 & 17,4 & 29,8 & 1,39 & 26,5 \\
.45 Automatic Colt Pistol & 12,1 & 17,0 & 29,1 & 1,41 & 26,5 \\
\hline
\end{tabular}


ainda estaríamos esperando a bala chegar ao ponto mais alto da trajetória com base nessa aproximação quando, na verdade, ela já estaria no chão!

Como podemos ver da Tabela 3 , considerando a resistência do ar, a altura máxima $h_{\max }$ atingida por todos os projéteis é da ordem de um $1 \mathrm{~km}$. Observe que os valores da altura máxima $h_{\max }$ são entre 3 e 7 vezes menores do que aqueles previstos nos casos em que a resistência do ar é desprezada $\left(h_{\text {Omax }}\right)$.

Os projéteis não ficam tempo o bastante em queda para atingirem a velocidade terminal $v_{t}$. Da Tabela 4 observamos que as velocidades de queda $v_{q}$ estão entre $91 \%$ e $98 \%$ do valor das velocidades terminais $v_{t}$ correspondentes.

Se no caso sem a resistência do ar, a energia mecânica é conservada, no caso em que a mesma é levada em conta, a situação é completamente distinta. A razão $\Delta E_{m} / k_{0}$ calculada para os projéteis nos informa que entre $82 \%$ e $96 \%$ da energia inicial do projétil é dissipada durante o trajeto (Tabela 4).

\subsection{A letalidade}

Comparando apenas as velocidades de saída $v_{0}$ e de queda dos projéteis $v_{q}$ (Tabela 4), poderíamos ser levados à conclusão de que é pouco provável a morte por projéteis que caem do céu. Ao observamos que o projétil quando chega ao solo pode ter apenas $4 \%$ da energia cinética inicial (.22 long rifle), essa conclusão pareceria ainda mais fortalecida. Contudo, essa é uma análise que ignora um ponto fundamental: a cabeça é o principal alvo de projéteis que caem.

Os projéteis atirados diretamente contra uma pessoa podem acertar uma grande área, o que inclui regiões vitais e não-vitais. A área que um projétil em queda vertical pode acertar uma pessoa é significativamente menor, mas possui a cabeça, uma região extremamente vital, como alvo mais provável (Fig, 3. De fato, em um estudo com 118 pacientes vítimas de projéteis em queda, verificou-se que $77 \%$ deles foram atingidos na cabeça. A taxa de mortalidade foi de $32 \%$, significativamente maior do que para todos os outros casos de ferimento por tiros em geral [8].

A velocidade em que um projétil é capaz de penetrar a pele encontra-se por volta de $45 \mathrm{~m} / \mathrm{s}$ a $60 \mathrm{~m} / \mathrm{s}$. Dessa forma, todos os projéteis analisados irão provocar esse efeito. Mais drástico do que isso é que projéteis com velocidade próximas de $60 \mathrm{~m} / \mathrm{s}$ já são capazes de fraturar ossos e mesmo penetrar no crânio [9]. Portanto, mesmo as armas de pequeno calibre analisadas podem ser letais para disparos verticais.

\section{Conclusão}

Determinamos as expressões para a posição $y(t)$, a velocidade $v(t)$, a altura máxima $h_{m} a x$ e o tempo de subida $t_{s}$ para o caso em que a resistência do ar é considerada. Os resultados obtidos são interessantes para se mostrar a diferença entre a situação com e sem a resistência do ar. As expressões obtidas podem ser utilizadas para se mostrar em sala de aula, por exemplo, até que ponto

Tabela 3: Altura máxima considerando a resistência do ar $h_{\max }$ e sem considerá-la $h_{O \max }$. A razão entre as alturas $h_{O \max } / h_{\max }$ é apresentada.

\begin{tabular}{lccc}
\hline Calibre & $h_{\max }(\mathbf{m})$ & $h_{\text {Omax }}(\mathbf{m})$ & $\frac{h_{\text {Omax }}}{h_{\max }}$ \\
\hline .22 short (5.6 mm short) & 705 & 4291 & 6,1 \\
.22 long rifle & 764 & 5556 & 7,3 \\
6.35 mm Browning & 743 & 2699 & 3,6 \\
7.65 mm Browning & 879 & 4592 & 5,2 \\
$9 \times 18$ mm Police & 704 & 2041 & 2,9 \\
7.62 mm Nagant & 884 & 4592 & 5,2 \\
.32 Smith and Wesson & 1109 & 4291 & 3,9 \\
.38 Smith and Wesson & 845 & 2250 & 2,7 \\
.38 Smith and Wesson special & 1072 & 3449 & 3,2 \\
.45 Automatic Colt Pistol & 1019 & 3449 & 3,4 \\
\hline
\end{tabular}

Tabela 4: Velocidade inicial $v_{0}$, de queda $v_{q}$ e terminal $v_{t}$ para diferentes projéteis. A razão $\Delta E_{m} / k_{0}$ e $v_{q} / v_{t}$ são apresentadas.

\begin{tabular}{lccccc}
\hline Calibre & $\begin{array}{c}v_{0} \\
(\mathrm{~m} / \mathrm{s})\end{array}$ & $\begin{array}{c}v_{q} \\
(\mathrm{~m} / \mathrm{s})\end{array}$ & $\frac{\left|\Delta E_{m}\right|}{k_{0}}$ & $\begin{array}{c}v_{t} \\
(\mathrm{~m} / \mathrm{s})\end{array}$ & $\frac{v_{q}}{v_{t}}$ \\
\hline .22 short (5.6 mm short) & 290 & 66,7 & 0,95 & 68,6 & 0,97 \\
.22 long rifle & 330 & 67,1 & 0,96 & 68,6 & 0,98 \\
6.35 mm Browning & 230 & 76,8 & 0,89 & 81,4 & 0,94 \\
7.65 mm Browning & 300 & 76,9 & 0,93 & 79,5 & 0,97 \\
9x18 mm Police & 200 & 79,3 & 0,84 & 86,4 & 0,92 \\
7.62 mm Nagant & 300 & 77,2 & 0,93 & 79,9 & 0,97 \\
.32 Smith and Wesson & 290 & 92,4 & 0,90 & 97,5 & 0,95 \\
.38 Smith and Wesson & 210 & 88,9 & 0,82 & 98,2 & 0,91 \\
.38 Smith and Wesson special & 260 & 95,2 & 0,87 & 102,2 & 0,93 \\
.45 Automatic Colt Pistol & 260 & 91,5 & 0,88 & 97,8 & 0,94 \\
\hline
\end{tabular}




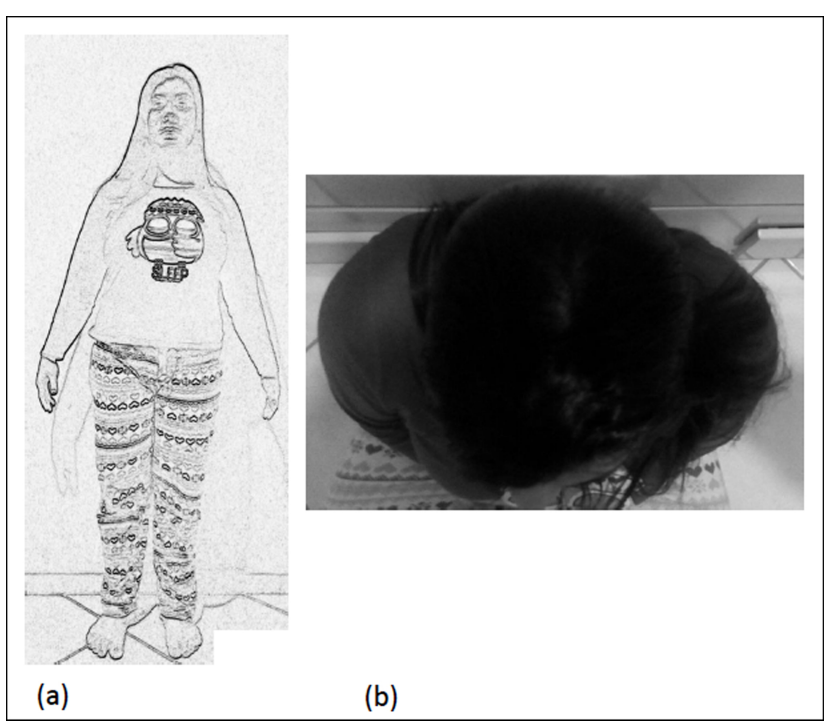

Figura 3: (a) Disparos em geral podem acertar regiões vitais ou não-vitais. (b) Quando em queda, a cabeça, região extremamente vital, torna-se o alvo mais provável.

a aproximação feita ao desprezar a resistência do ar é razoável.

A forma funcional consideravelmente mais complexa dessas expressões ilustra que, embora saibamos resolver o problema com a resistência do ar, o preço matemático que se paga é mais alto. Portanto, nas situações em que a aproximação de ignorarmos a resistência do ar for razoável, a facilidade de obtenção das soluções e análise das mesmas justifica o seu uso.

Para o nosso problema em específico, os resultados obtidos deixam claro que ignorar a resistência do ar não é uma opção; o valor das grandezas obtidas para os diversos projéteis destoam muito nos dois casos. Por exemplo, a energia dissipada que seria nula para o caso sem resistência do ar, chega a ser de $96 \%$ para o caso com a resistência do ar (.22 long rifle, Tabela 4).

Apesar da expressiva diminuição da velocidade dos projéteis analisados durante a queda, concluímos que a velocidade final $v_{q}$ ainda é suficiente para colocar em risco a vida das pessoas atingidas. Dessa forma, os nossos resultados justificam a Portaria Interministerial $\mathrm{n}^{\circ} 4.226$, de 31 de dezembro de 2010, que proibe os tiros de advertência pela Polícia Federal, Polícia Rodoviária Federal, Departamento Penitenciário Nacional e Força Nacional de Segurança Pública em razão da imprevisibilidade de seus efeitos. Não fosse o tiro para o alto potencialmente letal, mesmo aqueles de armas de pequeno calibre, tiros na vertical poderiam ser colocados como norma para tiros de advertência.

\section{Agradecimentos}

Os autores agradecem à Oficial da Força Aérea Brasileira Ana Lúcia Bezerra Cordeiro por ter chamado e, principalmente, despertado nossa atenção para este problema.

\section{Referências}

[1] S.A. Ali, S. M. Tahir, A. Makhdoom, A. R. Shaikh e A. R. Siddique, Iran. Red Crescent Med. J. 17, e26179 (2015).

[2] R. L. McCoy, Modern Exterior Ballistics (Schiffer Publishing, Atglen, 2001).

[3] C. I. Farrar e D. W. Leeming, Military Ballistics a Basic Manual, Battlefield Weapons Systems and Technology, vol.10 (Royal Military College of Science, Shrivenham, UK, 1983).

[4] D. G. Figueredo and A. F. Neves, "Equações Diferenciais Aplicadas," (IMPA, Rio de Janeiro, 2007).

55] M. S. D. Cattani, "Elementos de mecânica dos fluidos," (Blucher LTDA, São Paulo, 2005) $2^{\text {a }}$ ed.

[6] B. Madea, Handbook of Forensic Medicine, (WileyBlackwell, New Jersey, 2014).

[7] R. L. McCoy, Aerodynamic Characteristics of Caliber. 22 Long Rifle Match Ammunition (No. BRL-MR-3877), Army Ballistic Research Lab Aberdeen Proving Ground MD (1990).

[8] G. J. Ordog, P. Dornhoffer, G. Ackroyd, J. Wasserberger, M. Bishop, W. Shoemaker, e S. Balasubramanium, The Journal of trauma 37, 1003 (1994).

[9] A. N. Incorvaia, D. M. Poulos, R. N. Jones e J. M. Tschirhart, Ann. Thorac. Surg. 83, 283 (2007). 